\title{
VERIFICATION OF THE URICK-AMENT DESCRIPTION OF THE EFFECTIVE DYNAMIC RESPONSE OF RANDOM COMPOSITE FIBROUS MEDIA
}

\author{
A. WIRGIN and S. GHARIANI
}

Laboratoire de Modélisation en Mécanique, U.R.A. 229 du CNRS, Université Pierre et Marie Curie, 4 place Jussieu, F-75252 Paris cedex, France

Sound is often used to characterize the mechanical properties of heterogeneous materials. At very low frequencies it is relatively clear how to replace the composite medium by an effective (homogeneous) medium associated with a phase velocity which is some sort of average of the component phase velocities of the composite and an attenuation, if any, which is the sum of the attenuations of the various phases of the composite. At higher frequencies this prescription no longer applies since the observed attenuation and phase velocity vary with frequency (due to scattering) even when the component attenuations and phase velocities are independent of this parameter and the effective attenuation is usually not equal to the sum of the attenuations of the component materials.

The description of this (higher frequency) dispersive response is not evident. Nevertheless, Urick and Ament [1] were able to derive an exceedingly simple formula which relates the complex wavenumber of the effective medium (assumed to occupy a slab of arbitrary thickness) to the number of scatterers per unit volume within the slab, the wavenumbers in the scatterers and in the host medium, and to the far-field response of the individual scatterers (assumed to radiate as if they were struck individually by the probe radiation only) in the forward- and back-scattered directions corresponding to the directions of measured transmission and reflection. This formula, and its variants, have been submitted to surprisingly few verifications, probably because of the difficulty of producing well-characterized random solid composites with controlled volume fractions.

This difficulty can be circumvented by replacing the experiments by numerical simulations. We calculate the far-field response of a system of discrete scatterers located within a slab, and from this compute the phase velocity and attenuation which we then compare to the corresponding quantities arising from the Urick-Ament formula (modified to take into account the fact that spheres are replaced by cylinders). This program is based on an integral equation formulation including all inter and intra fiber interactions for up to twenty fibers and frequencies nearly exceeding the upper limit of the Rayleigh region (more fibers at lower frequency). The mutually parallel, identical fibers are elastic, located in an elastic host and the probe radiation is that of a shear horizontal wave. The numerical results were checked by comparison with calculations based on the cylindrical wavefunction expansion technique. The Urick-Ament formula is shown to give a good description of the response for low frequencies, small volume fractions of scatterers and small density and shear modulus contrasts between host and inclusions. 
Let a set of linear, homogeneous, isotropic, elastic (LHIE) mutually parallel, identical, randomly spaced cylinders (fibers) be contained within a LHIE slab (whose boundaries are not materialized) of arbitrary thickness and exposed to a shear wave associated with a displacement vector $u_{i}$ and propagation vector $\mathbf{k}_{1}$ which are parallel and perpendicular respectively to the fiber generators ( $z$ axis). With an implicit time factor exp(-iwt), $\omega$ the angular frequency, let $u_{i}(x)$ and $u(x)$ denote the z-component (the others being zero) of the incident and total displacement fields, with $x=(x, y)$. Assume the host material within and outside the slab to be $M_{1}$ and the fiber material to be $M_{2}$. The mass density and rigidity within $M_{j} ; j=1,2$ are denoted by $\rho_{j}$ and $\mu_{j}$ respectively. The shear phase velocity and wavenumber in $M_{j}$ are $\beta_{j}=\left(\mu_{j} / \rho_{j}\right)^{1 / 2}$ and $k_{j}=\omega / \beta_{j}$ respectively. Since we are assuming $M_{1}$ and $M_{2}$ to be non-lossy, $\beta_{j}$ and $k_{j}$ are real.

Normally speaking, it is not possible to speak of a well-defined wavenumber in a heterogeneous medium since $\mu$ and $\rho$ are not constants with respect to the space coordinates. Put in another way, no single plane wave can, by itself, propagate within the heterogeneous slab, since it is necessarily accompanied by scattered plane waves. Nevertheless, Urick and Ament [1] consider a slab filled

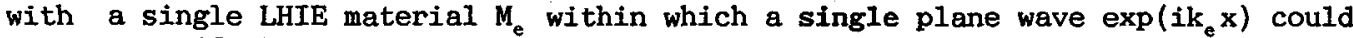
propagate if the medium were of infinite extent, and qualify this configuration (the effective medium) as being equivalent to the heterogeneous medium (within a slab) in the sense that both give rise to "the same reflected and transmitted waves" in response to an incident plane wave. This idea poses certain conceptual problems because it is not clear what is meant by "reflected and transmitted waves" in connection with a heterogeneous slab since the latter necessarily gives rise to scattering in both the reflection and transmission half-spaces. There is a problem even for homogeneous slabs, when, as is usually the case in experiments, the lateral extent of the slab is finite, thereby causing satellite waves to make their appearance (due to diffraction by the edges of the slab) around the directions of ordinary reflection and transmission (the same occurs for heterogeneous slabs as wel1). Waterman and True11 [2] were able to place the Urick and Ament idea on firmer theoretical ground and obtain the following result (which we [3] have extended to two-dimensional problems). The wavenumber $k_{e}$ in the effective medium is

$k_{e}(\omega)=k_{1}\left(\left[1+i \frac{2 N}{k_{1}^{2}} \mathscr{G}(0)\right]^{2}+\left[\frac{2 N}{k_{1}^{2}} \mathscr{C}(\pi)\right]^{2}\right) 1 / 2$.

where $N=\varphi / A$ is the number of fibers per unit area in the sagittal ( $x-y$ ) plane, $\varphi$ the filling factor (occupied area of the inclusions with respect to the total area of the slab in the sagittal plane), A the cross section area of the generic fiber, and $\mathscr{C}(\theta)$ the complex scattering function of a single isolated fiber in response to $u_{i}$. $\mathbb{E}(\theta)$ is related to the far-field scattered field of the fiber (composed of $M_{2}$ in a host of infinite extent filled with $M_{1}$ )

$u_{s}(x) \sim \mathscr{G}(\theta)\left(\frac{2}{\pi k_{1} r}\right)^{1 / 2} \exp \left[i\left(k_{1} r+3 \pi / 4\right)\right] ; k_{1} r \rightarrow \infty$,

with $\theta=0$ and $\theta=\pi$ the forward- and back-scattering directions respectively. In a weak-scattering regime, which occurs for small $\varphi$ and/or small contrast between the elastic constants of the host and the Inclusions, the second term in Eq. (1) is negligible compared to the first so that 
$k_{e}(\omega)=k_{1}\left[1+\frac{2 N}{k_{1}^{2}} G(0)\right]$.

Conservation of energy indicates that:

$\sigma_{e x t}=\sigma_{s c a}+\sigma_{A b s}=\frac{4}{k_{1}} \operatorname{Re}[G(0)]$.

with $\sigma_{\text {ext }}, \sigma_{\text {sa }}, \sigma_{\text {bs }}$ the cross-sections of extinction, scattering and absorption respectively for the isolated inclusion. From the fact that

$k_{m}=\omega / \beta_{m}+i \alpha_{m}=(\omega / \beta) n_{m}=(\omega / \beta)\left(n_{m}{ }^{\prime}+i n_{m}{ }^{\prime \prime}\right)=(\omega / \beta)\left[\left(\beta / \beta_{m}\right)+i\left(\beta \alpha_{m} / \omega\right)\right] ; m=1,2, e$,

( $\beta$ a real reference phase velocity) we finally obtain from Eqs. (3) and (4):

$\alpha_{e}(\omega)=(N / 2) \sigma_{e \times t}$.

$\beta_{e}(\omega)=\beta_{1}\left\{1-\left(2 N / k_{1}^{2}\right) \operatorname{Im}[G(0)]\right\}^{-1}$.

Eq. (6) is the principal result of the Urick-Ament, Waterman-Truell (UAWT) theory. It essentially expresses the facts that: 1) macroscopic scattering in the heterogeneous slab gives rise to dispersion $\left(\alpha_{e}\right.$ and $\beta_{e}$. depend on $\left.\omega\right), 2$ ) this dispersion is essentially governed by the density of scatterers and the forward scattering properties of the generic scatterer. As Beltzer and Brauner have pointed out [3], Eq. (6a) is actually the most important of the two since $\beta_{\mathrm{e}}(\omega)$ can (and should according to Beltzer and Brauner) be computed from $\alpha_{\mathrm{e}}(\omega)$ by means of the Kramers-Kronig (KK) relations. However, the KK computation changes radically the exceedingly simple nature of the UAWT result and should not be resorted to unless necessary [4].

To what extent can one rely on the UAWT to characterize the dynamic response of a heterogeneous material ? The more important question, on account of the possibility of resorting to the KK computation, is to what extent Eq. ( $6 \mathrm{a}$ ) is true? This equation is, in fact, a quite general consequence of the inherent assumptions: a) of a medium in which the positions of the scatterers are completely uncorrelated (i.e., fully random), and b) that each inclusion responds to the incident wave as if the other inclusions were absent. These assumptions evidently have their limits, which set in for large $\varphi$ and/or large elastic constants contrast. It is possible also that the frequency plays a major role. To examine these questions we resorted to numerical experiments which rely on the rigorous volume integral equation technique [5]. The cylindrical inclusions, which, for convenience, have square cross sections, are located within an imaginary slab and their positions are chosen by a random number generator for each realization. The response of the configuration to $u_{i}$ is computed with the necessary precision (necessitating an increasing number of field sampling points within each cylinder as the frequency, refractive index contrast and filling factor increase) for each realization and is repeated for a new realization from 10 to 50 times. The last step is to average (over the different realizations) the extinction coefficient of the $s$ lab, $C_{e x t}$, which is thought to be proportional to $\alpha_{e}$, and to average the global bistatic scattering cross section $|B(\theta)|^{2}$ of the slab. The response of the effective homogeneous slab (of same dimensions as that of the heterogeneous slab) is computed in the same manner and the choice of $\mathrm{k}_{\mathrm{e}}$ is made iteratively: first we use the static mixing formula approximation of $\mathrm{k}_{\mathrm{e}}$ [3]

$\alpha_{e 0}=\operatorname{Lim}(\omega \rightarrow 0) \alpha_{e}(\omega)=0, \beta_{e}=\operatorname{Lim}(\omega \rightarrow 0) \beta_{e}(\omega)=\left(n_{2}\right)^{1 / 2} \varphi+\left(n_{1}\right)^{1 / 2}(1-\varphi)$. 
then we do a simultaneous minimization of: a) the difference between the average extinction coefficient $\left\langle\mathrm{C}_{\mathrm{ext}}\right\rangle$ and the effective medium extinction coefficient, and $b)$ the difference between the average forward scattering cross section $\left\langle|B(0)|^{2}\right\rangle$ and the effective medium forward scattering cross section. Fig.1 exhibits the bistatic cross sections after averaging and for the optimized effective medium slab. The case treated is that in which $\mu_{1}=\mu_{2}$ and $\rho_{2}=2.25 \rho_{1}$. The homogeneous slab accounts for the rather high level of average diffuse component by the loss factor $\alpha_{e}$, the diffraction around the forward scattering direction being solely due to the finite extent of the slab. The UAWT gives exactly the same value for $k_{e}$ as the numerical experiment optimization technique for this rather low density of scatterers (although the frequency and refractive index contrast are not low).

Fig.2 concerns the test of Eq. (6a). The material constants are the same as in Fig.1. The UAWT predicts $\left\langle\mathrm{C}_{\mathrm{ext}}\right\rangle$ to be a linear function of $\varphi$, whereas the numerical experiments indicate rather a $\varphi^{1 \cdot 3}$ variation in the range considered. However, for very small $\varphi$, the variation is quasi linear. Thus the optimism generated by the result of Fig.1 is tempered by the present result. At present, we are carrying out the numerical experiments for larger $\omega$, $\varphi$ and refractive index contrasts.

[1] R.J. Urick and W.S. Ament, J.Acoust.Soc.Am. $21(1949) 115$.

[2] P.C. Waterman and R. Truel1, J.Math.Phys. 2(1961)512.

[3] A.I. Beltzer and N. Brauner, J.Mech.Phys.Solids 33(1985)471.

[4] A.I. Beltzer, S. Ghariani and A. Wirgin, J.Electromag.Waves Appl. (1992) (to appear)

[5] L. Rayleigh, Phil.Mag. 12(1881)81.

Fig.1 Average far-field response of a random heterogeneous slab and of its effective medium counterpart.

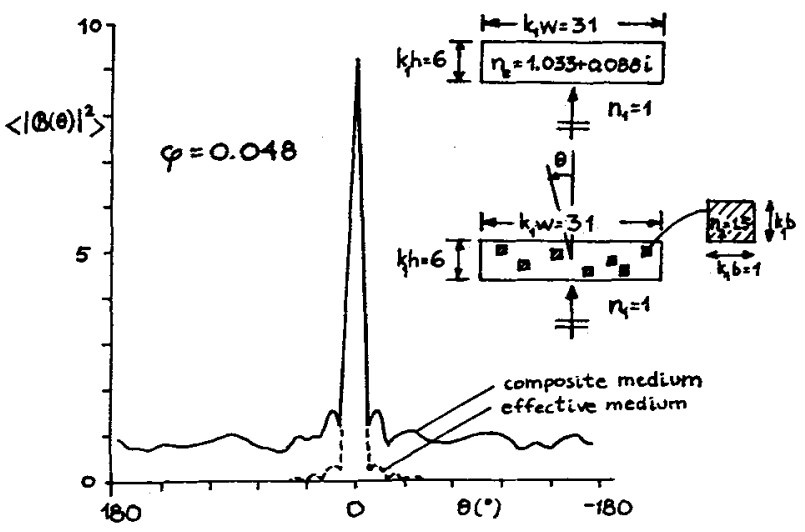

Fig.2 Average extinction cross section (for 50 realizations) versus filling factor for a random heterogeneous slab fitted to a power law. 